ORIGINAL ARTICLE

\title{
Comparison of heparin dressing and conventional dressing in second degree burn patients in term of pain relief and wound healing.
}

\author{
Iftikhar Alam¹, Abdul Malik Mujahid², Husnain Khan ${ }^{3}$
}

Article Citation: Alam I, Mujahid AM, Khan H. Comparison of heparin dressing and conventional dressing in second degree burn patients in term of pain relief and wound healing. Professional Med J 2022; 29(2):131-136. https://doi.org/10.29309/TPMJ/2022.29.02.6299

\begin{abstract}
Objective: To compare effectiveness of heparin soaked and conventional dressing in Second degree burn patients. Study Design: Randomized Controlled study. Setting: Department of Burn and Plastic Surgery, Teaching Hospital/ DG Khan Medical College. Period: November 2019 to October 2020. Material \& Methods: One hundred twenty patients fulfilling inclusion criteria were enrolled and randomly divided into two groups. Group A (60 patients) were treated with Heparin soaked Dressing and Group B (60 patients) with conventional dressing (Silver Sulphadiazine ointment $1 \%$ ) and were observed up to 3-weeks of post treatment. Outcome measures were noted and was assessed by Visual Analogue scale score for pain relief and complete re-epithelization of wound $(>75 \%)$ in the two groups. Significance was determined by comparing analgesia doses and total days required to heal in each dressing using chi-square test. Results: One hundred twenty patients were selected with mean age of patients was $14.4+7.4$ and male: female (62.5\%:37.5\%). Mean TBSA\% was $12 \pm 1.80$. Mean pain score in the group A for Superficial partial thickness burn (SPTB) and Deep dermal burn was $4 \pm 1$ and $6 \pm 1$ respectively ( $P$-value $<0.001$ ). Total Number of days for re-epithelization in the Group A and Group B (SPTB $13 \pm 1$ vs. 18 measures in term of pain relief and re-epithelization. Total analgesia requirement in the Group A and Group B (50 \pm 20 vs. $116 \pm 12 \mathrm{mg}$; P-value $<0.000$ for Superficial Partial Thickness burn and $46 \pm 6$ vs. $126 \pm 12 \mathrm{mg}$; P-value $<0.001$ for Deep partial thickness burn) respectively. There was significant difference in Outcome. Conclusion: Heparin soaked dressing is more useful and effective in Second degree burn in term of pain relief and re-epithelization in early post burn period compared to the conventional dressing.
\end{abstract}

Key words: $\quad$ Second Degree Burn, Topical Heparin, Visual Analogue Score, Re-epithelization, Silver Sulphadiazine 1\% Ointment, Pain Relief.

\section{INTRODUCTION}

Burn injury represents a significant burden of acute trauma for both conservative and surgical management that may require half of injured patients to get hospital admission. ${ }^{1}$ Improvement in Burn management has resulted in large no of survivors but long term disabilities can occur as a result of their skin and soft tissue injury that may need different treatment modalities including scar management, physical and occupational therapy sessions and reconstructive surgery. An average total health care cost is approximately $\$ 90000$ in middle and high-income countries per patient as evidenced in recent review of literature. ${ }^{2}$
When a cutaneous thermal injury occurs, changes occur in its composition. These include protein denaturation, cell lysis resulting in cytokines release such as Tumour necrosis factor alpha, IL-1 and IL-8. This inflammatory y cascade results in loss of endothelial vascular integrity, complement activation and leukocytes degranulation, local cellular apoptosis. ${ }^{3}$

It may be difficult to accurately assess the depth of burn wounds. Depth of burn wounds is that necessitate more than one method of local wound care. To expedite wound healing it may be important to have rapid and effective treatment of burnt skin. In ancient time for that
1. MBBS, FCPS (Plastic Surgery), Senior Registrar Plastic Surgery, DG Khan Medical College/Teaching Hospital Dera Ghaz Khan.

2. MBBS, FCPS (Plastic Surgery), Assistant Professor Plastic Surgery, DG Khan Medical College/Teaching Hospital Dera Ghazi Khan.

3. MBBS, FCPS (Plastic Surgery), Assistant Professor Plastic Surgery, , Rawalpindi Medical University /Holy Family Hospital Rawalpindi.

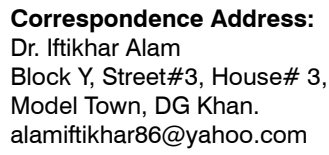

Correspondence Address:

Dr. Iftikhar Alam

Block Y, Street\#3, House\# 3,

Model Town, DG Khan.

alamiftikhar86@yahoo.com

Article received on:

$28 / 12 / 2020$

Accepted for publication: 
purpose application of dressings with the goals of keeping wound moist, promoting reepithelialization, avoiding water and heat loss preventing infection and decreasing pain were used. A variety of dressing have been used that include conventional, embryological, biological, Nonadherent films or fine mesh gauze with antibiotic coated are common dressings used to cover the burn wound, but films, foams, alginates, hydrocolloids, and hydrogels can also be used depending upon specific needs of the burn wound. Partial-thickness burns have moderate-to-high amounts of exudate, which are appropriately managed with foams and alginates. To cover clean, uninfected burn wounds variety of biologic dressings or skin substitutes such as human amnion, allograft skin and xenograft. The low frequency of dressing change and easy application make these dressings practical but drawback of these biological dressings includes high cost, availability, transmission of infection and storage. ${ }^{4}$

Aggressive wound care that includes topical agents with antimicrobial activity has been associated with reduced incidence of invasive wound infections. A large systematic review included 56 trials with over 5800 mostly adult patients with partial-thickness burns involving less than $40 \%$ TBSA. Various studies compared a given topical agent or dressing with silver sulfadiazine (SSD) ointment. ${ }^{5}$

Other comparisons included alternative silver based agents or dressings, iodine-based agents, chlorhexidine, polyhexanide, sodium hypochlorite, ethacridine lactate, cerium nitrate, honey, aloe Vera, Polysporin is a combination of bacitracin zinc and polymyxin Bsulfate for the partial-thickness burns. All of these dressing have potential drawback of inadequate Wound healing with pseudo-eschar formation and incomplete pain relief.

Heparin dressing a novel agent is being used to relieve pain and facilitate rapid epithelization. Burn studies have revealed that heparin has antiinflammatory, anti-allergenic, anti-histaminic and other enzymatic properties. It has been used in topical forms in the management of thermal burn to prevent extension of burn area, hasten healing with fewer contractures, relieve of pain, reduce tissue edema and to promote revascularization, and re-epithelialization of burn tissue. Earlier in 2011, a study revealed that topical heparin is effective in management of partial thickness burn (Ghana study) in burn management in term of pain relief and wound healing.

In another Study by M Masoud et al, 35\% patients of conventional group received analgesia twice daily in the second week of treatment while Topical Heparin treated patients received analgesics on demand after 2nd week onwards. Hospital stay was 18.3 days in the Conventional group, as compared to Topical heparin treatment group who had an average hospital stay of 12.3 days $(P<0.05) .{ }^{6}$ In another Study Number of days for wound healing were significantly lower in heparin group (for both SPTB and DPTB $4 \pm 1$ vs. $6 \pm 1$; P-value $<0.000$ ) than conventional group (SPTB $13 \pm 1$ vs. $18 \pm 4$ days; P-value $<0.000$ and for DPTB, $15 \pm 2$ vs. $19 \pm 2$ days; P-value $<0.003$ ).

The rationale of this study was to determine the frequency of pain relief and re-epithelization of 2nd degree burn with the use of heparin soaked dressing versus conventional dressing (Silver Sulphadiazine 1\% ointment). As no local study with large sample size is available in literature, so the results of this study will help in change of clinic practice and will add in evidence-based practice for partial thickness burn management.

\section{MATERIAL \& METHODS}

This Study was conducted at Burn and Plastic Surgery Unit, Teaching Hospital, DG Khan Medical college, DG Khan from November 2019 to October 2020 The sample size of 120 patients was calculated with 95\% confidence interval and margin of error $5 \%$ and taking expected \%age of pain relief $90 \%$ of heparin soaked dressing in $2^{\text {nd }}$ degree burn. Patients full filling inclusion criteria (either gender, age range 8 to 60 years and wound with thermal burns on front of chest, abdomen, upper limb and lower limb up to 35 $\%$ body surface area, Superficial partial, deep partial thickness) were selected through non- 
probability consecutive sampling. Randomly divided into two groups. Group A (60 patients) were treated with Heparin soaked Dressing and Group B (60 patients) with conventional dressing (Silver Sulphadiazine ointment 1\%) Patients with allergy to heparin, coagulopathies, patients with liver disease. Hypertension, diabetes determined via medical record and laboratory analysis were excluded from study.

After the approval from the ethical review board of the hospital, written informed consent was obtained from all the patients. After baseline investigations, pre-treatment photography was done for the record purpose. All patients underwent heparin soaked dressing. All patients were followed up weekly up to 3-week. Daily dressing of wound was done for each group. A total $20 \mathrm{ml}$ of $5000 \mathrm{IU} / \mathrm{ml}$ of heparin solution was added to $250 \mathrm{ml}$ of normal saline solution and a total $270 \mathrm{ml}$ of $400 \mathrm{IU} / \mathrm{ml}$ heparinized solution was prepared. The heparinized solution was sprinkled, twice daily on sterile gauze, starting at day 1 . Heparin in decreasing quantity was continued until final healing. For $15 \%$ of burn surface area on day 1, Total heparin dose was $100,000-120,000$ IU.

Approximately, $75 \%$ of day 1 dose was used on the burn wound surfaces on day 2nd, 3rd and 4th $50 \%$ of day 1 dose was from day 5 th onward. Burn blisters were treated with hypodermic needle with syringe, filled with $150-200 \mathrm{IU} / \mathrm{ml}$ heparin. Small puncture was made with needle, blister fluid was drained. The blister was then rinsed slowly with heparin 2-3 times, and then the needle withdrawn, residual volume of heparin was left within the blister. The outer surface of blister was permitted to settle onto the blister's inner surface. Blisters were not debrided or removed.

\begin{tabular}{|c|c|c|c|c|c|c|c|}
\hline & \multicolumn{2}{|c|}{ Group A (H) $(n=60)$} & \multicolumn{2}{|c|}{ Group B (C) $(n=60)$} & \multicolumn{2}{|c|}{ Total $(n=120)$} & \multirow{3}{*}{ P-Value } \\
\hline & Frequency & Percentage & Frequency & Percentage & Frequency & Percentage & \\
\hline Age Mean \pm SD & \multicolumn{2}{|c|}{$33.0 \pm 10.19$} & \multicolumn{2}{|c|}{$33.73 \pm 9.55$} & \multicolumn{2}{|c|}{$14.37 \pm 9.80$} & \\
\hline $15-35$ & 42 & 70 & 38 & 63.33 & 80 & 66.7 & \multirow{2}{*}{$P=.440$} \\
\hline $36-50$ & 18 & 30 & 22 & 36.66 & 40 & 33.3 & \\
\hline \multicolumn{8}{|l|}{ Gender } \\
\hline Male & 38 & 60.3 & 37 & 64.9 & 75 & 62.5 & \multirow{2}{*}{$P=0.605$} \\
\hline Female & 25 & 49.7 & 20 & 35.1 & 45 & 37.5 & \\
\hline \multicolumn{8}{|l|}{ Burn cause } \\
\hline Flame & 18 & 30.0 & 17 & 28.4 & 35 & 29.2 & \multirow{3}{*}{$P=0.966$} \\
\hline Flash & 12 & 20.0 & 13 & 21.6 & 25 & 20.8 & \\
\hline Scald & 30 & 50.0 & 30 & 50.0 & 60 & 50.0 & \\
\hline \multicolumn{8}{|l|}{ Degree of burn } \\
\hline $\begin{array}{l}2^{\text {ND }} \text { DEGREE } \\
\text { SUPERFICIAL }\end{array}$ & 35 & 60.0 & 36 & 60.0 & 71 & 59.2 & \multirow[t]{2}{*}{$P=0.432$} \\
\hline $2^{\mathrm{ND}}$ DEGREE DEEP & 25 & 40.0 & 34 & 40.0 & 59 & 40.8 & \\
\hline \multicolumn{8}{|c|}{ Time to Epithelization } \\
\hline$\leq 14$ days & 43 & 73.4 & 33 & 66.67 & 76 & 70.0 & \multirow{2}{*}{$P=0.059$} \\
\hline$>14-28$ days & 17 & 26.6 & 27 & 33.33 & 44 & 30.0 & \\
\hline \multicolumn{8}{|c|}{$\begin{array}{l}\text { Analgesia requirement } \\
\text { (first week) }\end{array}$} \\
\hline On Demand & 12 & 20.0 & 8 & 13.4 & 20 & 16.7 & \multirow{4}{*}{$P=0.396$} \\
\hline Once a day & 16 & 26.6 & 12 & 20.0 & 28 & 23.3 & \\
\hline Twice a day & 16 & 26.6 & 16 & 26.6 & 32 & 26.7 & \\
\hline Three times a day & 16 & 26.6 & 24 & 40.0 & 40 & 33.3 & \\
\hline
\end{tabular}




\begin{tabular}{|l|c|c|c|}
\hline \multicolumn{1}{|c|}{ Variable } & Group A (H) $\mathbf{( n = 6 0 )}$ & Group B (C) (n=60) & P-Value \\
\hline Pain score & $11.5+\ldots 1.6$ & $15.4+\_1.45$ & 0.001 \\
\hline Hospital stay (days) & $15.73 \pm 2.89$ & $22.8 \pm 4.44$ & 0.001 \\
\hline
\end{tabular}

Table-II. Pain score and hospital stay among groups

Both groups were evaluated for need of total analgesics and healing time. Heparin treatment was also standardized and the outcome measures i.e. pain relief and re-epithelization (epithelialization was measured as the percentage of the wound with a vital skin cover as compared to normal skin on clinical assessment). were noted on specially designed Performa. Data was entered and analysed using SPSS 22. For the quantitative variables like the age, the size of wound and pain (Visual Analogue Scale) mean and standard deviation was calculated. Qualitative variables like gender, pain relief and re-epithelization were calculated in the form of frequencies and percentages. Data was stratified for age, gender and the size of the wound. The post stratified chi-square test was applied to see role of effect modifiers. P-value $\leq 0.05$ was considered as significant.

\section{RESULTS}

A total of 120 cases were enrolled. The mean age of patients was $14 \pm 10.00$ years. (Table-I) Gender distribution of patients showed that there were $74 \%(62)$ females and $46 \%$ (38) male patients included in the study. (Table-I). Mean $\%$ TBSA of burn was $12 \pm 1.80 \mathrm{~cm}$. Minimum and maximum \%TBSA was $5 \%$ and $35 \%$ respectively. (Table-I). At the $5^{\text {th }}$ day, 74(62\%) patients had pain relief (the VAS score of 3 or less) (Table-I,II) and 94(78\%) patients had achieved re-epithelialization (Table-II) The pain relief at the 5th day was not significantly associated with the age group of patients. (Table-I).

\section{DISCUSSION}

Different type of dressing are used in $2^{\text {nd }}$ degree burn which are individualized based on the condition and depth of burn wound. Research process continues for a safe, reliable, cost effective treatment of burn. The aim of such dressings is pain relief, prevention of wound desiccation, reduce trauma, promote epithelization and to control bacterial growth. The Conventional daily dressing with $1 \%$ silver sulfadiazine is suitable for evolving burn wound or if there is need of daily mechanical debridement for pseudo eschar, eschar, or necrotic burn tissue.

Slow release Silver dressings are customized in different ways and are widely available but they have disadvantage of yellow discolouration and necrosis of normal healthy tissue. ${ }^{7}$

Pain at the 2nd degree burn site can be a real problem for most patients especially in first five post burn days. The use of topical Heparin is in practice in burn patient since many years. It has neoangiogenic, collagen-restoring antiinflammatory, and epithelializing effects in addition to its anticoagulation properties. Use of topical heparin and its safety has been well documented in literature. ${ }^{8}$ Topical heparin solution has advantage over dry dressing both in terms of healing and pain management in 2 nd degree burn. In multiple studies, use of heparin topically in second degree burn has shown very encouraging results as far as the pain relief is concerned. In those patients who completed the study, Analgesic efficacy was evaluated by Visual Analog Scale (VAS) for pain relief. Result of this study showed that analgesic requirement was lower in patients treated with topical heparin $(11.83 \pm 9.38$ per patient against $33.35 \pm 20.63$ for the conventional dressing, $p<0.01$ ), and has low VAS score for pain. ${ }^{9}$ There was statistically significant difference for heparin group both for pain relief and epithelialization.

With regard to hospital stay the Study showed, $35 \%$ of Heparin group patient had a hospital stay of 1 week, whereas in the Control group, the same percentage of patients stayed for around 2 weeks $\left(P<0.05\right.$.Agate benorku et $\mathrm{al}^{10,11}$, have reported that the total hospital stay of patients during admission was reduced in the Heparin group. There was statistically significant difference in mean hospital stay of Heparin dressing patients 
compared with-group (silver Sulphadiazine and or Polyfax skin ointment) in ten to twenty percent burns (13 vs. 26 days). This study also compared the use of Heparinized soaked dressing with conventional dressing for pain relief in second degree burn. This study demonstrated that when burn wound site dressing was kept moist through the instillation of aqueous solution of diluted heparin with the help of a catheter placed in it, it produced considerably more pain relief. This considerably reduced the need for on demand analgesia compared to conventional dressing.

In another Study by M Masoud et al, 35\% patients of conventional group received analgesia twice daily in the second week of treatment while Topical Heparin group received analgesics on demand after the $2^{\text {nd }}$ week. Hospital stay was 18.3 days in the Conventional group as compared to Topical heparin group who had an average hospital stay of 12.3 days $(P<0.05) .{ }^{12}$ In another Study Number of days for wound healing were significantly lower in topical heparin $(4 \pm 1$ vs. $6 \pm 1$; P-value $<0.000)$ than conventional group (SPTB $13 \pm 1$ vs. $18 \pm 4$ days; P-value $<0.000$ and for DPTB, $15 \pm 2$ vs. $19 \pm 2$ days; $P$-value $<0.003) .{ }^{13,14,15}$

In our study, Mean pain score in the group was $4 \pm 1$ and $6 \pm 1$ respectively ( $P$-value $<0.000$ ). Duration of re-epithelization in the group $A$ and group $B$ was (SPTB $13 \pm 1$ vs. $18 \pm 4$ days; $p$-value $<0.000$ and for DPTB, $15 \pm 2$ vs. $19 \pm 2$ days; P-value <0.003) respectively. Total analgesia requirement in the Group A and Group B $(50 \pm 20$ vs. $116 \pm 12 \mathrm{mg}$; $p$-value $<0.000$ for SPTB and $46 \pm 6$ vs. $126 \pm 12 \mathrm{mg}$; P-value $<0.000$ for DPTB) respectively. Pain relief and epithelization were effectively controlled with Topical heparin dressing.

Limitation of this study is that this is single centre study which limits its uniformity. Another limitation is that the conventional treatment included only topical silver Sulphadiazine has been shown repeatedly to impede wound healing with delayed wound healing in conventional group than the accelerated healing observed in heparin group.

\section{CONCLUSION}

Heparin soaked dressing is more useful and effective in Second degree burn in term of pain relief and re-epithelization in early post burn period compared to the conventional dressing.

\section{Copyright $@ 14$ June, 2021.}

\section{REFERENCES}

1. American Burn Association. Burn incidence fact sheet. https://ameriburn.org/who-we-are/media/burnincidence-fact-sheet/. Accessed August 31, 2018.

2. Hop MJ, Polinder S, van der Vlies $\mathrm{CH}$, Middelkoop E, van Baar ME. Costs of burn care: A systematic review. Wound repair and regeneration. $2014 \mathrm{Jul}$; 22(4):436-50.

3. Lantz M, Thysell H, Nilsson E, Olsson I. On the binding of tumor necrosis factor (TNF) to heparin and the release in vivo of the TNF-binding protein I by heparin. The Journal of clinical investigation. $1991 \mathrm{Dec}$ 1; 88(6):2026-31

4. Gupta, A., Gupta, P. \& Verghese, T. Role of topical heparin in the management of burns: Experience in a district government hospital of Karnataka in South India. Plastic and Aesthetic Research, 2015; 2(3), 111.

5. Masoud M, Wani AH, Darzi MA. Topical heparin versus conventional treatment in acute burns: a comparative study. Indian Journal of Burns. 2014 Jan 1; 22(1):43.

6. Saliba Jr MJ. Heparin in the treatment of burns: A review. Burns. 2001 Jun 1; 27(4):349-58.

7. Wasiak J, Cleland H, Campbell F, Spinks A. Dressings for superficial and partial thickness burns. Cochrane Database of Systematic Reviews. 2013(3). https://doi. org/10.1002/14651858.CD002106.pub4.

8. Dr Sanand Prem, et al. "Role of Topical heparin in Management of Burns". IOSR Journal of Dental and Medical Sciences (IOSR-JDMS), 19(2), 2020, pp. 25-27. DOI: $10.9790 / 0853-1902082527$.

9. Hamza FA, Salim AA, Mohammed HN. Evaluation of the Effect of topical heparin on the treatment of facial burn. The Egyptian Journal of Hospital Medicine. 2019 Oct $1 ; 77(4): 5455-60$.

10. Jan, Saadia Nosheen, Farid Ahmed Khan, Muhammad Mustehsan Bashir, Muneeb Nasir, Hamid Hussain Ansari, Hussan Birkhez Shami, Umer Nazir, Asif Hanif, and Muhammad Sohail. "Comparison of Laser Doppler Imaging (LDI) and clinical assessment in differentiating between superficial and deep partial thickness burn wounds." Burns 44, no. 2 (2018): 405413. doi:http://dx.doi.org/10.1016/j.burns.2017.08.020. 
11. Agbenorku P, Fugar S, Akpaloo J, Hoyte-Williams PE, Alhassan Z, Agyei F. Management of severe burn injuries with topical heparin: The first evidencebased study in Ghana. International journal of burns and trauma. 2013; 3(1):30.

12. Manzoor, S., Khan, F. A., Muhammad, S., et, al., Comparative study of conventional and topical heparin treatment in second degree burn patients for burn analgesia and wound healing. Burns, 2019; 45(2),379-386. doi.org/10.1016/j.burns.2018.05.0.

13. Bharathi SM, Sudhakar PV. Role of topical heparin therapy in thermal burns. International Journal of Contemporary Medical Research ISSN. 2017; 4:2235-9.
14. Alfeky $H$, McArthur $P$, Helmy $Y$. Salvaging digital replantation and revascularisation: efficiency of heparin solution subcutaneous injection. Surgery research and practice. 2018 Nov 21; 2018.

15. Barretto MG, Costa MD, Serra MC, Afiune JB, Praxedes HE, Pagani E. Comparative study of conventional and topical heparin treatments for burns analgesia. Journal of the Brazilian Medical Association. 2010; 56(1):51-5.

\begin{tabular}{|c|c|c|c|}
\hline \multicolumn{4}{|c|}{ AUTHORSHIP AND CONTRIBUTION DECLARATION } \\
\hline No. & Author(s) Full Name & Contribution to the paper & Author(s) Signature \\
\hline 1 & Iftikhar Alam & $\begin{array}{l}\text { Principal contributor, Concep- } \\
\text { tualization and design of re- } \\
\text { search work, Data collection, } \\
\text { Writing of manuscript statsiti- } \\
\text { cal, Analysis. }\end{array}$ & 慗 \\
\hline 2 & Abdul Malik Mujahid & $\begin{array}{l}\text { Drafting, review of results and } \\
\text { final approval. }\end{array}$ & \\
\hline 3 & & $\begin{array}{l}\text { Statistical analysis, Interpreta- } \\
\text { tion of data, Results analysis, } \\
\text { Literature search, final review. }\end{array}$ & $\mathbb{H}$ \\
\hline
\end{tabular}

